\title{
Barriers to ESC guideline implementation, results of a survey from the European Council on Cardiovascular Nursing and Allied Professions (CCNAP)
}

Gabrielle McKee $^{a, b}$, Mary Kerins ${ }^{a, c}$, Glenys Hamilton ${ }^{a, d, e}$, Tina Hansen ${ }^{a, f}$, Jeroen Hendriks $a, g, h$, Eleni Kletsiou ${ }^{a, i}$, Ekaterini Lambrinou ${ }^{a, j}$, Catriona Jennings ${ }^{a, k}$, Donna Fitzsimons ${ }^{a, l}$.

${ }^{a}$ Council on Cardiovascular Nursing and Allied Health Professionals (CCNAP), European Society of Cardiology, The European Heart House, Les Templiers, 2035 Route des Colles, CS 80179 BIOT, 06903 SOPHIA ANTIPOLIS Cedex, France.

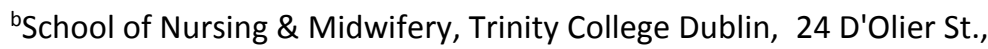
Dublin D02 T283, Ireland. gmckee@tcd.ie

${ }^{c}$ Cardiac rehabilitation, St James Hospital, James St, Dublin 8, Ireland. mkerins@stjames.ie

${ }^{d}$ Consultant in Nursing Education and Cardiovascular Research, 601 George Hill Road, Lancaster, MA. 01523, USA.

eMassachusetts General Hospital, 55 Fruit Street, Boston, MA. USA, 02114 glenys@mac.com.

fDepartment of Cardiology; Zealand University Hospital, Hospitalsvej 10, 4000 Roskilde, Denmark. tbh@regionsjaelland.dk

${ }^{g}$ Centre for Heart Rhythm Disorders, University of Adelaide, Royal Adelaide Hospital, South Australian Health and Medical Research Institute, Adelaide, Australia North Terrace, Adelaide, SA 5000, Australia.

hDepartment of Health and Medical Sciences, Linköping University, Linköping, Sweden Campus US, 58183 Linköping, Sweden. jeroen.hendriks@adelaide.edu.au

'University General Hospital "ATTIKON", Chaidari, Athens, Greece

Rimini 1, PC 12462, Chaidari, Greece. eklets@nurs.uoa.gr

jDepartment of Nursing, School of Health Sciences, Cyprus University of Technology, 15, Vragadinou str, 3041 Limassol, Cyprus. ekaterini.lambrinou@cut.ac.cy

kDepartment of Cardiovascular Medicine, National Heart and Lung Institute, Imperial College London, Hammersmith Hospital Campus, ICTEM Building, London W12 0NN, England.

c.jennings@imperial.ac.uk

'School of Nursing, Queens University, Belfast, N Ireland. d.fitzsimons@ulster.ac.uk

Corresponding author:

Gabrielle McKee,

School of Nursing \& Midwifery, Trinity College Dublin, 24 D'Olier St., Dublin D02 T283 , Ireland

Phone: 0035318962779

Fax: 0035318963001

Email: gmckee@tcd.ie 


\section{Barriers to guideline implementation, results of a survey from the European Council on Cardiovascular Nursing and Allied Professions (CCNAP)}

\section{Abstract}

Background: The European Society of Cardiology (ESC) has a comprehensive clinical guideline development programme, relevant for all clinicians. However, implementation of guidelines is not always optimal.

Aim: The aim of this study was to determine nurses' and allied professionals' awareness and barriers regarding clinical guideline implementation.

Methods: A cross-sectional survey was administrated online and in print at EuroHeartCare 2015. A questionnaire was developed which examined awareness and barriers to implementation of ESC Guidelines on cardiovascular disease prevention in clinical practice (2012) and ESC guidelines in general.

Results: Of the 298 respondents, $12 \%$ reported that the prevention guidelines were used in their practice area. Respondents identified, in order of magnitude, that lack of leadership, workload, time, resources and a perception that they were unable to influence current practice, were barriers to the use of the prevention guidelines. When asked to rank barriers to use of any ESC guidelines, time (22\%) and leadership (23\%) were ranked highest.

Conclusions: Implementation of ESC Guidelines by nurses, the majority responders in this survey, is a serious problem, requiring urgent improvement to ensure patients receive optimal evidence based care. Issues of leadership, workload, time and resources are significant barriers to guideline implementation. It is of concern that these professionals perceive both that they have little influence on implementation decisions and lack of leadership regarding guideline implementation. Educational and organisational strategies to improve leadership skills are imperative. These will build self-efficacy and empower nurses and allied professionals to advocate for evidence based care in the clinical environment. 
Key words: clinical guidelines, implementation, barriers, prevention, cardiac.

\section{Implications for Practice:}

For respondents in this study the main implications for practice were:

- Nurses and all health professionals require clinical leadership and seniors to demonstrate good role modelling to serve as best practice examples in guideline use and implementation

- The guidelines are complex and thus require implementation strategies which employ the whole multidisciplinary team including physicians, nurses and other allied health professionals all playing their role

- Guideline implementation is an integral part of care and should not be seen as an optional extra to care duties

\section{Introduction:}

Despite advances in treatment and care, cardiovascular disease remains the leading cause of death within Europe, accounting for $42 \%$ of all mortality in $2014 .{ }^{1}$ Up to date treatment and care are guided by research evidence. Clinical guidelines, such as those developed by the European Society of cardiology (ESC), synthesise the vast range of evidence available to clinicians and simplify it into clear-cut recommendations for practice. Despite the development of guidelines and their known effectiveness, there are many barriers to optimal implementation and consequently the achievement of optimal patient outcomes is compromised. Most of the cardiovascular research conducted has focused on barriers to guideline implementation in physicians, particularly in the primary care sector, with less research in nursing or allied health professionals..$^{2-8}$

Barriers to guideline implementation have been categorised into three main groups; issues with the guidelines themselves, professional and personal factors and issues within the organizational and environmental context. ${ }^{8}$ 
Issues with the guidelines themselves have been frequently reported and include lack of guideline awareness, lack of familiarity and disagreement with content. ${ }^{2,8-13}$ In addition, issues such as guideline complexity, length, unavailability of translations and accessibility have also been widely reported. ${ }^{4-6,11-12,14,}$ Professionals' attitude and proficiency also influence effective guideline implementation. Examples of these barriers include lack of training and skills, knowledge, motivation and the opinion that implementation restricts autonomous decision making. ${ }^{3-5}$ 10,13-It is al There are also patient related barriers to implementation. Some clinicians are of the opinion that guidelines are theoretically based and not practically oriented and have concerns about patient adherence and acceptability ${ }^{2,-5,10-12,14-15}$ In addition, clinicians themselves report being overloaded with too many guidelines. ${ }^{5,9,11,14}$ Work related obstacles are also numerous and varied and include time constraints, poor support, leadership and team collaboration, lack of resources including financial constraints, and incompatibility with workplace policies. ${ }^{2,4-5,9-13,15}$ External factors were also implicated and one study found that the most important barrier to implementation was government health policy. ${ }^{15}$ Given the interdisciplinary arena in which cardiovascular care is practiced, nurses and allied professionals have an important role in contributing to the development and implementation of guidelines. The ESC Council on Cardiovascular Nursing \& Allied Professions (CCNAP) sought to determine members' knowledge of and attitudes towards guideline implementation and use these data to support their educational initiative: "Be Guideline Smart", which is designed to support and empower nurses to work in an interdisciplinary way to ensure implementation of guidelines in clinical practice. The above synthesis of the literature indicates the complexity of the task of improving guideline implementation.

Clearly, improving guideline implementation is a multi-faceted clinical issue, and simple solutions are unlikely to succeed. Greater insight into the specific factors relevant to cardiovascular care should enable the development of appropriate multidisciplinary strategies to improve guideline implementation and as a consequence impact on patient outcomes. The aim of this study was to determine awareness and barriers to the use and implementation of both, ESC prevention clinical 
guidelines and other ESC guidelines in general, in nurses and allied professionals working with cardiac patients.

\section{Methods:}

The study used a cross-sectional survey design. Ethical approval for this study was obtained from the establishment of the lead investigator (School of Nursing and Midwifery, Trinity College Dublin Research Ethics Committee) and return of the questionnaire was taken as consent. The investigation conforms to the principles outlined in the Declaration of Helsinki (Br Med J 1964; ii: 177).

Data collection, sample and population: Data were collected by an anonymous questionnaire during the period April 2015-September 2015. The inclusion criteria for the study were nurses or allied health professions working with cardiac patients. The sampling frame included CCNAP members, non-member visitors to CCNAP and national society's websites and attendees at the EuroHeartCare Conference 2015. Members of CCNAP received an e-mail inviting them to participate in the study. The link to the survey was embedded in the e-mail and also posted on the CCNAP website, where it was available in several languages. In order to increase the number of responses, members were also encouraged to pass the link on to their colleagues. In addition, the mail was forwarded through the National Society Committee members to distribute within their national societies. Finally, attendees at the EuroHeartCare conference 2015 in Dubrovnik, had the opportunity to complete a paper copy of the questionnaire on site if they had not previously completed it online. A follow-up reminder message was sent to all CCNAP members and posted on the website.

The above recruitment strategy utilised a non-probability convenience sample method. There was no stratification within the sampling and therefore the sample cannot assume to represent a stratified sample of all nurses and allied health professionals working with cardiac patients or the membership of CCNAP. In order to insure that the sample was numerically sufficient to represent 
the sampling frame post hoc sample size calculation was performed. For a key outcome, knowledge of any ESC guidelines, $25 \%$ of the sample rated their knowledge as very good or excellent. The CCNAP membership is approximately 2200 nurses and allied health professionals. For this sampling frame size, this prevalence of very good/excellent knowledge and a confidence level of $95 \%$, a sample size of 289 would be required to reflect the knowledge of the sample frame. ${ }^{16}$

Data collection tools: A questionnaire was developed by the researchers based on the literature and included 16 questions in total, including one twenty eight part question on barriers to the utilisation of prevention guidelines and one eight part question ranking barriers to the use of any ESC guidelines (difficulty, access, length, time, financial incentive, leadership, not in a position of influence guideline implementation and do not have the confidence to challenge other members of the multidisciplinary team). There were three main sections: sociodemographic questions, selfreported use and the perceived barriers to the use of the Fifth Joint Task Force European Guidelines on Cardiovascular Disease prevention in Clinical Practice (2012) and knowledge and ranked barriers to the use of any ESC guidelines. ${ }^{17}$ Whilst face validity of the questionnaire was examined by the research team, construct validity was assessed by eight external experts, two academics with cardiac expertise, six hospital clinical staff with up to 15 years cardiac experience, currently working in chest pain, heart failure, rehabilitation and the catheterization laboratory. They completed Likert format questions to assess both clarity and relevance and also had the opportunity to add free text comments. A total of $85 \%$ of questions, instructions or statements were returned, with $70 \%$ of respondents indicating that they were very clear or very relevant. The $15 \%$ of questions, instructions or statements that did not meet this standard and any comments presented by the reviewers were reviewed by the research team and the survey revised accordingly.

Analysis: Descriptive statistics were used to analyse the data. In order to examine the relationship between eleven respondents' personal profile characteristics (gender, age, geographical region, profession, years of cardiac experience, current position, highest education level, self-rated 
knowledge, recent reading of guidelines, preferred formats, use of prevention guidelines in practice), use of prevention guidelines in their practice and their ranking of time and leadership as barriers to their self-reported knowledge of any ESC guidelines, Spearman's rho and logistic regression was used. The significance level was set at 0.05 . SPSS, version 23 was used for the statistical analysis.

\section{Results:}

There were 298 completed questionnaires returned from 37 different countries, of which $86 \%$ were submitted online. The majority of the respondents were female (82\%), nurses (86\%) and $58 \%$ of the respondents were employed in academic teaching hospitals. The most common area of expertise was heart failure (48\%) and $83 \%$ had a qualification of degree or higher of which $45 \%$ had a Master's degree (Table 1).

Twelve percent of respondents indicated that the ESC prevention guidelines were routinely used in their current practice areas (Data not shown in tables). Of those who reported guidelines other than ESC guidelines being used, $9 \%$ (28) of respondents named these, with $6 \%(18)$ reporting that they used national guidelines. Several respondents who reported not using any guidelines took the opportunity to comment on their reasons with free text: "Place of nurse in the guidelines in my country is not defined", "Nurses do as the doctor tells them and hopefully our doctors are using updated guidelines".

Just over half of respondents identified the pocket guidelines as their preferred format for the prevention guidelines (Figure 1). When respondents were asked about the last time they had accessed the prevention guidelines, $(n=284), 53 \%$ in total had accessed them within the last 3 months (Figure 2). 
Table 2 shows the perceived barriers to implementation of the prevention guidelines. The vast majority of respondents rated the prevention guidelines as good educational tools (94\%), easy to use $(88 \%)$ and easy to access (82\%). However, $40 \%$ reported that they are too long, and $40 \%$ indicated that they are too rigid to apply to individual patients. Seventy one percent of the respondents reported that their clinical teams consistently believe in the importance of guidelines and $58 \%$ reported a lack of leadership for guideline implementation in their clinical practice areas. This was in addition to identifying that workload, insufficient resources and not enough time prevented them from adequately implementing and following up the recommendations in the guidelines. Although a large proportion of respondents believe they have a responsibility to use guidelines, a significant proportion reported having educational needs $(66 \%)$ and a perceived lack of ability to influence current practice decisions (37\%).

Just over a third of the respondents (37\%) rated their knowledge of any ESC guidelines as very good or excellent. In the question examining the ranked barriers to any ESC guideline use, leadership $(23 \%)$ and time (22\%) were the barriers that received the highest proportion of first ranking (Figure 3). Bivariate analyses of the 12 variables and knowledge of any ESC guidelines, found significant associations with four variables, nationality $\left(\chi^{2}(1)=6.58, p=0.037\right)$, a higher level of education $\left(\chi^{2}(1)=.142, p=0.015\right)$, prevention guidelines used in their practice $\left(\chi^{2}(1)=7.64, p=0.02\right)$, and a more recent reading of the guidelines $\left(\chi^{2}(1)=17.38, p<0.001\right)$. Logistic regression was used to test if these variables significantly influenced their self-rated knowledge of any ESC guidelines. The model was significant $\left(\chi^{2}(11, N=270)=30.443, p=0.002\right)$. High self-rated knowledge of any ESC guidelines was associated with older age, non-European nationality and having recently read the prevention guidelines (Table 3).

Bivariate analyses of the 12 variables and citing leadership as the highest ranking barrier found significant associations with three variables, age $\left(\chi^{2}(1)=6.66, p=0.01\right)$, prevention guidelines used in their practice $\left(\chi^{2}(2)=12.58, p=0.002\right)$ and a more recent reading of the prevention guidelines 
$\left(\chi^{2}(1)=9.38, p=0.002\right)$. Logistic regression was used to test if these variables significantly influenced their rating leadership as the greatest barrier to any ESC guideline implementation. Three factors were found to be significantly associated with citing leadership as a barrier: younger age, working in a hospital and prevention guidelines were not used in their practice areas $\left(\chi^{2}(10, N=270)=31.50\right.$, $p<0.001)$. (Table 4). Age had the highest odds ratio in logistic regression analyses with younger respondents 2.4 times more likely to cite leadership as a barrier to any guideline implementation (Table 4).

\section{Discussion:}

This study is the first to investigate guidelines utilisation and barriers to their implementation in nurses and allied health professionals working with cardiac patients across diverse European regions where the standard of education and the standing of nurses varies enormously. This cohort reported a lack of leadership in the clinical environment, a perception of being unable to influence current practice, a lack of resources such as time and staffing, and related workload as the main barriers to the use and implementation of guidelines.

Firstly looking at the use and implementation of prevention guidelines. In contrast to the literature, where guideline implementation rates were $58 \%$ to $80 \%$, this study found a low reported use $(12 \%)$ of the ESC prevention guidelines in this cohort although $53 \%$ had consulted them in the last three months. ${ }^{9,12,18}$ Nearly half of the respondents reported working in heart failure and may only use their specialty guidelines in their patient care. However, the high recent consultation of the prevention guidelines may indicate that if some of the barriers to guideline implementation were lessened these professionals would be equipped to engage even further with their patients regarding general prevention in cardiac disease and facilitate every contact counting regarding prevention. While previous research indicates clinicians working in European countries more often use national and local guidelines, in this study irrespective of their speciality few respondents 
indicated that they used alternative guidelines. ${ }^{11}$ In support of previous research, the main barriers to the implementation and use of prevention guidelines were a lack of leadership, inadequate resources insufficient time, workload and perception that they were unable to influence current practice. $^{4-5,8,10,12-13}$

Secondly looking at the knowledge and use of any ESC guidelines, a similar picture is revealed. Only $37 \%$ of respondents perceived their general knowledge of any ESC guidelines to be excellent or very good, which is low in contrast to other studies, albeit in most of these studies knowledge of guidelines was assessed objectively (51\%-91\%). ${ }^{5}$ Using logistic regression this study found that selfrated knowledge of any ESC guidelines was lowest in the younger respondents, those from Western Europe and those who had not read the ESC prevention guidelines recently. While in comparison to previous studies, which used bivariate analyses only, gender, profession, years of cardiac experience, current position, education level, main source of information on risk factors and the preferred format of guidelines were not significant influences on self-rated knowledge of any ESC guidelines. ${ }^{5}$ This study supports previous research in physicians but also in nurses and allied health professionals suggesting that time is a major issue in the implementation of both the prevention and other published guidelines..$^{2-6,8-9.11-13,15}$ Time may encompass other barriers also examined such as resources and workload.

The barriers and problems highlighted in this study in part reflect the difficulty of improving guideline implementation. The ESC Guidelines for prevention in contrast to those for the management of acute myocardial infarction in patients presenting with ST-segment elevation implementation do not include detailed pathways and algorithms. ${ }^{19,20}$ We could presume that detailed pathways may make implementation easier as they are known across the team etc., but there is no evidence of this. In addition, attitudes to certain guidelines may play a part, it has been observed that prevention guideline implementation may be perceived as an optional extra by respondents in the pressurised care environment, rather than an integral part of care. ${ }^{21}$ These 
findings highlight the need for the development of implementation strategies to approach prevention at an organisational as well as a team level.

Although level of education in this study did not have a direct effect on self-rated knowledge of any guidelines, providing information not only on guidelines content, but incorporating implementation strategies which utilise cooperative learning and team teaching, as advocated in the CCNAP core postgraduate curriculum may also assist in better guideline implementation. This would facilitate a truly multidisciplinary teamwork approach to guidelines implementation. ${ }^{22}$

This study supports previous work that effective guideline implementation requires leadership and an organisational culture that supports nurses to advocate for guideline based care..$^{5}$ Lack of leadership has been more frequently reported in nursing compared to physician studies..$^{5,10,12}$ In addition this study found that younger age, working in hospital and a low use of prevention guidelines in clinical practice areas were associated with citing leadership as the greatest barrier to guideline implementation of any ESC guideline. It is a concern that younger nurses perceive a lack of clinical leadership in relation to guideline implementation. As pointed out by Abrahamson and colleagues, when nurses see physicians not supporting guidelines use, it acts as a real barrier to their own ability to do so. ${ }^{10}$ With an increasing number of studies demonstrating that nurse led initiatives and case management approaches in cardiac care are effective and the ESC supporting the development of nurses as equal partners with clinicians in the European Society of Cardiology as Guideline Task Force members, reviewers, and active members of ESC Boards and Committees, there is no longer any excuse for nurses to lag behind. ${ }^{23-30}$ The time to address these issues is now, with many opportunities presenting themselves, such as the CCNAP "Be Guideline Smart" campaign which has seen the development of a range of resources to assist nurses and health professionals to become more aware and knowledgeable about guidelines.

[https://www.escardio.org/Councils/Council-on-Cardiovascular-Nursing-and-Allied-Professions(CCNAP)/Council-on-Cardiovascular-Nursing-and-Allied-Professions-CCNAP, accessed 21 Sep 2016]. In addition, a tool kit is available utilising typical clinical scenarios where nurses demonstrate tackling 
common issues in clinical practice to help nurses to address some of the barriers identified in this study. It also includes links to the ESC Guideline resources. We anticipate that increased awareness, knowledge and skills gained from the use of these resources will empower and assist nurses and allied health professionals to instigate local initiatives to address some of the barriers identified in this study.

Piepoli and the Sixth Joint Task Force on Cardiovascular Prevention in the 2016 edition of the ESC prevention guidelines states that the acute care team should emphasise the importance of preventive measures to the patient and ensure that prevention strategies are sustained and supported in other settings. ${ }^{20}$ CCNAP anticipates that knowing the specific barriers to guideline use will help facilitate the development of effective educational, organisational and team strategies that will improve guideline implementation and consequently patient outcomes, a development in which nurses, in particular, are in the ideal position to play a major role at many levels. ${ }^{20}$

\section{Limitations:}

The method of recruitment, mainly via the CCNAP website, and the non-probability sampling method - convenience sampling without stratification, caused a bias that resulted in the respondents being most frequently nurses, masters educated, working in academic hospitals and with expertise in heart failure and or acute care. This may limit the generalisability of the results as the sample can neither assume to represent a stratified sample of all nurses and allied health professionals working with cardiac patients. The small proportion of allied health professionals as respondents made it unfeasible to analyse the results of this cohort separately. The study utilised self-report and not objective measures of guideline implementation therefore as with all self-report studies there is an increased risk of a more positive desirable response. ${ }^{31}$ However, this method is the most common method used in the research cited. In addition, an anonymous questionnaire was used which should reduce this risk. 


\section{Conclusion}

Nurses and allied professions do not utilise guidelines as effectively as they should, potentially compromising the quality of patient care. We have identified that issues related to leadership, time, resources and workload are the greatest barriers to guideline implementation. Effective strategies to educate and empower the whole multidisciplinary team using a cooperative approach need to be developed to address these barriers to implementation. Nurses have a professional responsibility to become more involved in guideline implementation and are in an ideal position to advocate for patients and facilitate the delivery of guideline based care.

\section{Acknowledgments:}

The study group would like to thank the following for their support: CCNAP National associations committee members for distributing the link to their national committees; Prof Christi Deaton and Prof Tiny Jaarsma for their work in the development of the Be Guideline Smart campaign and Novartis for their continued support of the Be Guideline Smart campaign; the EURObservational Research Programme (EORP) for developing the online version of the questionnaire and the initial analysis. This research received no specific grant from any funding agency in the public, commercial, or not-for-profit sectors. 


\section{References}

1. Nichols M, Townsend N, Scarborough P and Rayner M. Cardiovascular disease in Europe 2014: epidemiological update. Eur Heart J. 2014; 35: 2950-9.

2. Schmieder RE, Goebel M and Bramlage P. Barriers to cardiovascular risk prevention and management in Germany-an analysis of the EURIKA study. Vasc Health Risk Manag. 2012; 8 : 177.

3. Brotons C, Lobos JM, Royo-Bordonada MA, et al. Implementation of Spanish adaptation of the European guidelines on cardiovascular disease prevention in primary care. BMC Fam Pract. 2013; 14: 36

4. Abrahamson KA, Fox RL and Doebbeling BN. Facilitators and barriers to clinical practice guideline use among nurses. Am J Nurs. 2012; 112: 26-35.

5. Jun J, Kovner CT and Stimpfel AW. Barriers and facilitators of nurses' use of clinical practice guidelines: An integrative review. Int J Nurs Stud. 2016; 60: 54-68.

6. Lee PY, Liew SM, Abdullah A, et al. Healthcare professionals' and policy makers' views on implementing a clinical practice guideline of hypertension management: a qualitative study. PLoS One. 2015; 10: e0126191.

7. Hakkennes $\mathrm{S}$ and Dodd K. Guideline implementation in allied health professions: a systematic review of the literature. Qual Saf Health Care. 2008; 17: 296-300.

8. Ploeg J, Davies B, Edwards N, Gifford W and Miller PE. Factors influencing best-practice guideline implementation: lessons learned from administrators, nursing staff, and project leaders. Worldviews Evid Based Nurs. 2007; 4: 210-9.

9. Dallongeville J, Banegas JR, Tubach F, et al. Survey of physicians' practices in the control of cardiovascular risk factors: the EURIKA study. Eur. J. Prev. Cardiol. 2012; 19: 541-50.

10. Ferrante D, Konfino J, Linetzky B, Tambussi A and Laspiur S. Barriers to prevention of cardiovascular disease in primary care settings in Argentina. Rev Panam Salud Publica. 2013; 33: 259-66. 
11. Hobbs FD and Erhardt L. Acceptance of guideline recommendations and perceived implementation of coronary heart disease prevention among primary care physicians in five European countries: the Reassessing European Attitudes about Cardiovascular Treatment (REACT) survey. Fam. Pract. 2002; 19: 596-604.

12. Erhardt L, Komajda M, Hobbs FDR and Soler-Soler J. Cardiologists' awareness and perceptions of guidelines for chronic heart failure. The ADDress your Heart survey. Eur J Heart Fail. 2008; 10: $1020-5$

13. Hadely KA, Power E and O'Halloran R. Speech pathologists' experiences with stroke clinical practice guidelines and the barriers and facilitators influencing their use: a national descriptive study. BMC Health Serv Res. 2014; 14: 1-41.

14. Reiner Z, Sonicki Z and Tedeschi-Reiner E. Physicians' perception, knowledge and awareness of cardiovascular risk factors and adherence to prevention guidelines: the PERCRO-DOC survey. Atherosclerosis. 2010; 213: 598-603.

15. Graham IM, Stewart M, Hertog MGL and Force obotCRTT. Factors impeding the implementation of cardiovascular prevention guidelines: findings from a survey conducted by the European Society of Cardiology. Eur J Cardiovasc Prev Rehabil. 2006; 13: 839-45.

16. Naing L, Winn T and Rusli BN. Practical Issues in Calculating the Sample Size for Prevalence Studies. 2006.

17. Perk J, De Backer G, Gohlke H, et al. European Guidelines on cardiovascular disease prevention in clinical practice (version 2012). The Fifth Joint Task Force of the European Society of Cardiology and Other Societies on Cardiovascular Disease Prevention in Clinical Practice. Eur Heart J. 2012; 33: 1635-701.

18. Mosca L, Linfante AH, Benjamin EJ, et al. National study of physician awareness and adherence to cardiovascular disease prevention guidelines. Circulation. 2005; 111: 499-510. 
19. Steg PG, James SK, Atar D, et al. ESC Guidelines for the management of acute myocardial infarction in patients presenting with ST-segment elevation. Eur Heart J. 2012; 33: 2569-619.

20. Piepoli MF, Hoes AW, Agewall S, et al. 2016 European Guidelines on cardiovascular disease prevention in clinical practice. 2016. Eur Heart J. 2016; 37:2315-81.

21. Powell-Cope GM, Luther S, Neugaard B, Vara J and Nelson A. Provider-perceived barriers and facilitators for ischaemic heart disease (IHD) guideline adherence. J Eval Clin Pract. 2004; 10: 227-39.

22. Astin F, Carroll DL, Ruppar T, et al. A core curriculum for the continuing professional development of nurses: Developed by the Education Committee on behalf of the Council on Cardiovascular Nursing and Allied Professions of the ESC. Eur J Cardiovasc Nurs. 2015; 14 : 190-7.

23. Wood DA, Kotseva K, Connolly S, et al. Nurse-coordinated multidisciplinary, family-based cardiovascular disease prevention programme (EUROACTION) for patients with coronary heart disease and asymptomatic individuals at high risk of cardiovascular disease: a paired, clusterrandomised controlled trial. The Lancet. 2008; 371: 1999-2012.

24. Jennings C, Kotseva K, De Bacquer D, et al. Effectiveness of a preventive cardiology programme for high CVD risk persistent smokers: the EUROACTION PLUS varenicline trial. Eur Heart J. 2014; 35: 1411-20.

25. Jorstad HT, von Birgelen C, Alings AM, et al. Effect of a nurse-coordinated prevention programme on cardiovascular risk after an acute coronary syndrome: main results of the RESPONSE randomised trial. Heart. 2013; 99: 1421-30.

26. Ylimaki EL, Kanste $\mathrm{O}$, Heikkinen $\mathrm{H}$, Bloigu R and Kyngas $\mathrm{H}$. The effects of a counselling intervention on lifestyle change in people at risk of cardiovascular disease. Eur J Cardiovasc Nurs. 2015; 14: 153-61. 
27. Janssen V, Gucht VD, Dusseldorp E and Maes S. Lifestyle modification programmes for patients with coronary heart disease: a systematic review and meta-analysis of randomized controlled trials. Eur. J. Prev. Cardiol. 2013; 20: 620-40.

28. Berra K, Miller NH and Jennings $\mathrm{C}$. Nurse-based models for cardiovascular disease prevention: from research to clinical practice. Eur J Cardiovasc Nurs. 2011; 10 Suppl 2: S42-50.

29. Deaton C. Implementing clinical practice guidelines: a responsibility for nurses and allied health professionals? Eur J Cardiovasc Nurs. 2012;11(3): 263-264,

30. Berra K. Does nurse case management improve implementation of guidelines for cardiovascular disease risk reduction? J Cardiovasc Nurs. 2011; 26: 145-67.

31. Adams AS, Soumerai SB, Lomas J and Ross-Degnan D. Evidence of self-report bias in assessing adherence to guidelines. Int J Qual Health Care. 1999; 11: 187-92. 
Table 1. Participant profile $(n=298)$

\begin{tabular}{|c|c|c|}
\hline Profile variables & Subdivisions & $\%(n)$ \\
\hline \multirow[t]{2}{*}{ Gender } & Male & $17.8 \%(53)$ \\
\hline & Female & $82.2 \%(245)$ \\
\hline \multirow[t]{2}{*}{ Age (years) } & $\leq 45$ & $46.6 \%(139)$ \\
\hline & $>45$ & $53.4 \%(159)$ \\
\hline \multirow[t]{3}{*}{ Nationality } & Western Europe & $72.5 \%(216)$ \\
\hline & Eastern Europe & $19.8 \%(59)$ \\
\hline & Other & $7.7 \%(23)$ \\
\hline \multirow[t]{2}{*}{ Profession } & Nurse & $85.9 \%(256)$ \\
\hline & Other allied health professionals & $14.1 \%(42)$ \\
\hline \multirow[t]{2}{*}{ Years of Cardiac experience } & $\leq 10$ years & $29.9 \%(89)$ \\
\hline & $>10$ years & $70.1 \%(209)$ \\
\hline \multirow[t]{2}{*}{ Current position } & Academic teaching hospital & $58.4 \%(174)$ \\
\hline & Other & $41.6 \%(124)$ \\
\hline \multirow[t]{9}{*}{ Area of expertise } & Heart Failure & $48.3 \%(144)$ \\
\hline & General cardiology & $47 \%(140)$ \\
\hline & Acute cardiac care & $31.2 \%(93)$ \\
\hline & Arrhythmias & $29.9 \%(89)$ \\
\hline & Cardiac rehabilitation & $26.2 \%(78)$ \\
\hline & Catheterization laboratory & $16.4 \%(49)$ \\
\hline & Surgery & $15.4 \%(46)$ \\
\hline & Interventional cardiology & $13.4 \%(40)$ \\
\hline & Other & $9 \%(33)$ \\
\hline \multirow[t]{2}{*}{ Education } & Certificate or diploma & $18.1 \%(54)$ \\
\hline & Degree or higher & $81.9 \%$ (244) \\
\hline
\end{tabular}


Table 2: Perceptions about and barriers to implementation and use of the Fifth Task Force European Guidelines on Cardiovascular Disease Prevention in Clinical practice (version 2012) $(n=296)$

\begin{tabular}{|c|c|}
\hline & $\begin{array}{l}\text { Strongly agree / } \\
\text { Agree }\end{array}$ \\
\hline \multicolumn{2}{|l|}{ Issues with the guidelines themselves } \\
\hline The guidelines are good educational tools & $93.9 \%(278)$ \\
\hline The guidelines are easy to use & $88.2 \%(261)$ \\
\hline The guidelines are easy to access & $82.1 \%(243)$ \\
\hline Guidelines are too rigid to apply to individual patients & $40.4 \%(119)$ \\
\hline The guidelines are too long & $40.2 \%(119)$ \\
\hline There are inconsistent messages from different guidelines & $35.8 \%(106)$ \\
\hline The guidelines change too often & $18.9 \%(56)$ \\
\hline \multicolumn{2}{|l|}{ Operational issues with the guidelines } \\
\hline There is a consistent message throughout the team that guidelines are important $(n=294)$ & $71.4 \%(210)$ \\
\hline $\begin{array}{l}\text { Although risk factors are monitored usual care in my practice area does not always have } \\
\text { automatic follow up }(n=295)\end{array}$ & $69.2 \%(204)$ \\
\hline There is no leadership on guideline implementation in my workplace & $57.8 \%(171)$ \\
\hline $\begin{array}{l}\text { There are not enough resources to adequately implement and follow up the recommendations } \\
\text { in the guidelines }\end{array}$ & $57.4 \%(170)$ \\
\hline Lack of time is a major barrier to using guidelines & $53.4 \%(158)$ \\
\hline There is a cost incurred in using the guidelines & $27 \%(80)$ \\
\hline There is a financial incentive to use guidelines as part of patient care in my practice area $(n=295)$ & $20.7 \%(61)$ \\
\hline \multicolumn{2}{|l|}{ Personal perceptions and issues with guidelines } \\
\hline I feel I have the responsibility to use the guidelines & $92.5 \%(274)$ \\
\hline There should be patient versions of the guidelines $(n=295)$ & $90.5 \%(267)$ \\
\hline The guidelines help patients set appropriate goals ( $n=295)$ & $85.8 \%(253)$ \\
\hline Patients should be involved in guideline development & $81.7 \%(242)$ \\
\hline I would need further information on guideline updates & $76 \%(225)$ \\
\hline $\begin{array}{l}\text { I have the confidence to challenge other members of the multidisciplinary team when guidelines } \\
\text { are not implemented }\end{array}$ & $68.2 \%(202)$ \\
\hline I would need further education to implement the guidelines & $65.9 \%(195)$ \\
\hline I understand all the detail in the guidelines & $65.2 \%(193)$ \\
\hline $\begin{array}{l}\text { My workload prevents me taking on the additional task of ensuring guidelines are implemented } \\
\text { and followed up }\end{array}$ & $48.4 \%(143)$ \\
\hline $\begin{array}{l}\text { I am not in a position to influence current practices and routines that would assist the } \\
\text { implementation of guidelines }\end{array}$ & $36.6 \%(109)$ \\
\hline The guidelines have unrealistic targets for patients to achieve & $21.3 \%(63)$ \\
\hline I do not agree with some of the guidelines $(n=295)$ & $20 \%(59)$ \\
\hline I do not use the guidelines because my role does not specify that I should & $18.6 \%(55)$ \\
\hline
\end{tabular}


Table 3: Logistic regression analysis significant associations between self-rated knowledge of any European Society of Cardiology guidelines and participants profile.

\begin{tabular}{|c|c|c|c|c|c|}
\hline & & \multirow{2}{*}{$p$} & \multirow{2}{*}{ Odds ratio } & \multicolumn{2}{|c|}{ 95\% Confidence interval } \\
\hline & & & & Upper & Lower \\
\hline \multirow[b]{2}{*}{ Last read guidelines } & Within the last year & \multirow[b]{2}{*}{$<0.001^{*}$} & \multirow[b]{2}{*}{0.243} & \multirow[b]{2}{*}{0.107} & \multirow[b]{2}{*}{0.549} \\
\hline & More than a year ago & & & & \\
\hline \multirow{2}{*}{ Age } & $\leq 45$ years & \multirow{2}{*}{$0.037^{*}$} & \multirow{2}{*}{1.950} & \multirow{2}{*}{1.042} & \multirow{2}{*}{3.651} \\
\hline & $>45$ years & & & & \\
\hline \multirow{3}{*}{ Country } & Western European & \multirow{3}{*}{$0.007^{*}$} & \multirow{3}{*}{1.474} & \multirow{3}{*}{1.112} & \multirow{3}{*}{1.955} \\
\hline & Eastern European & & & & \\
\hline & Other & & & & \\
\hline
\end{tabular}

* Significant at $p<0.05$ 
Table 4: Logistic regression analysis significant associations between citing leadership as the main barrier to use of any ESC guidelines and participants profile and use of guidelines.

\begin{tabular}{|c|c|c|c|c|c|}
\hline & & \multirow{2}{*}{$p$} & \multirow{2}{*}{ Odds ratio } & \multicolumn{2}{|c|}{ 95\% Confidence intervals } \\
\hline & & & & Upper & Lower \\
\hline \multirow{3}{*}{ Age } & $\leq 45$ years & \multirow{3}{*}{$.018^{*}$} & \multirow{3}{*}{2.432} & \multirow{3}{*}{1.161} & \multirow{3}{*}{5.094} \\
\hline & & & & & \\
\hline & $>45$ years & & & & \\
\hline \multirow{2}{*}{ Current position } & Hospital & \multirow{2}{*}{$.023^{*}$} & \multirow{2}{*}{3.055} & \multirow{2}{*}{1.165} & \multirow{2}{*}{8.014} \\
\hline & Other & & & & \\
\hline \multirow{3}{*}{$\begin{array}{l}\text { Are the prevention guidelines } \\
\text { used in your practice }\end{array}$} & Yes & \multirow{3}{*}{$.005^{*}$} & \multirow{3}{*}{.434} & \multirow{3}{*}{.242} & \multirow{3}{*}{.777} \\
\hline & No & & & & \\
\hline & Unsure & & & & \\
\hline
\end{tabular}

* Significant at $\mathrm{p}<0.05$ 